$\xi=$ 줄

\title{
Assessment of occupational burnout among private dental practioners in Bengaluru city -a cross sectional study
}

\author{
MayurNath Reddy *, Shwetha HL, Mayur Nandikshor Mishra \\ Vydehi institute of Dental Science and research centre, Dept of public health dentistry, \\ \#82 EPIP AREA, Nellurahalli, whitefield, Bangalore 560066 \\ *Corresponding author E-mail:Mayurnm21@gmail.com
}

\begin{abstract}
Introduction: Dentistry is highly rewarding profession, but it's very demanding job with high degree of concentration and precision in work. Dentists seem to be prone to professional burnout, anxiety disorders and clinical depression because of the variety of sources of stress encountered throughout the professional career.

Objectives: To assess the burnout level and physical exhaustion among private dental practitioners.

Materials and methods: A cross sectional study was carried out on private dental practitioners in Bengaluru city. A questionnaire containing 22 items Maslach Burnout Inventory and demographic detail, was administered. The response rates were obtained using a likert scale ranging from $0-6$. Analysis for $p$ value, cronbach's $\alpha$ for each questions $(>0.700=$ high consistency), frequency and comparison of variables, were done.

Results: The study found high level of burnout in $11.3 \%$ of participants. It also showed age group of $<=25$ and $25-35$, females were highly influenced. More amount of burnout level found in general practitioners than specialists. Study also indicates that backache $48 \%$ and headache $44.7 \%$ were main reasons for physical exhaustion followed byneck-ache $34 \%$ and hand and wrist disorders $23.7 \%$. Conclusion: As health care provider the dentists are subjected to interpersonal stress due to the demanding nature of the occupation and close proximity to the patient.Moderate to high level of burnout were detected in the study sample, it's recommended that attention for burnout risk should be given priority by dental professionals.
\end{abstract}

\section{Introduction}

The term burnout was introduced in the 1970s by Maslach and Leiter, and was defined as a syndrome with emotional exhaustion (EE), depersonalization (DP) and diminished personal accomplishment (PA) that can occur among individuals working with people (Galán F et al 2013, p.1).

One of the possible consequences of chronic work-related stress is professional burnout. Professional burnout consists of three defining dimensions: emotional exhaustion, the development of a negative or cynical attitude towards one's patients or clients [depersonalization or cynicism], and the tendency to evaluate oneself negatively [diminished personal accomplishment. Although physical exhaustion may also be a sign of burnout, it is the feeling of being emotionally depleted that is considered the core element of professional burnout (Gorter RC et al 2012, p.87).

Dentists seem to be prone to professional burnout, anxiety disorders and clinical depression because of the variety of sources of stress encountered throughout the professional career, and there is the possibility of beginning as early as university (Galán $\mathrm{F}$ et al2013, p.1).The stress resulting from the relationship between the dentist as helper and patient as recipient is an important factor in the development of burnout. According to Leiter and Schaufeli burnout presents in professions characterized by intense and direct contact with others (Basson RA 2012, p.168). The dentists are part of a profession which is characterized by showing great working hours and has direct and daily contact with patients, who are many times tense and anxious regarding to the treatment they would be submitted, because there is an idea that the dental treatment causes pain (Zini A et al 2013,p.138).

As health care provider the dentist is subject to interpersonal stressors due to the demanding nature of the occupation and close proximity to the patient. Work-stress and long working hours may have a negative effect on the dentist's psychological well being and family life. Peterson et al.Study on service workers (including dentists) showed an association between burnout and depression, anxiety, alcohol consumption, sleep and memory problems as well as musculoskeletal complaints (Basson RA 2012, p.168).The ignorance of the burnout risk may contribute to the negative implications for the dentist, patient, and the work quality. A burned out dentist may be subject to accuse from the professional community as a professional who is not strong enough to carry on doing what has to be done. An over stressed dentist today might burnout in the long run.

The continuous exposure to stress inducers may cause depressive symptoms. Burnout is considered as an occupational health issue, one of the most important work related problems in today's society (Kapoor S et al 2014, p.17).Hence, it is paramount important to assess occupational burnout among private dental practitioners in Bengaluru city. 


\section{AIM}

To assess occupational burnout among private dental practitioners in Bengaluru city.

\section{Methodology}

A cross sectional study is carried out among the private dental practitioners in Bengaluru city. The study was done for a duration of one month in September-October, 2015. A cross sectional study was carried out among the sample of 150 IDA registered dentists taken according to stratified random sampling, after dividing Bengaluru city in 2 zones (north, and south arbitrarily) and based on inclusion exclusion criteria.

\subsection{Inclusion criteria}

- IDA registered private practicing dentists from Bangalore city.

\subsection{Exclusion criteria}

- Dentists who did not give consent for the study.

Organization and Administration Workout

1) Ethical clearance: The study proposal was approved by institutional review board of Vydehi Institute of Dental Sciences and Research centre, Bangalore.

2) Informed consent: The purpose and details of the study was explained to the study participants and a written consent was then obtained from them.

\subsection{Pilot study}

A pilot study was done among 40 privately practicing dentists of Vydehi institute of dental science and research centre, to check the feasibility and to validate the questionnaire. Face validity of the questionnaire was checked by asking experts to scrutinize the questions, while content validity was checked by ensuring that the questions covered all the areas of knowledge mapped out by initial objective. The reliability of the questionnaire was assessed by using Cronbach's $\alpha$ and it was found to be $>0.7$ (acceptable). Sample Size was obtained after Calculation based on the current pilot study.

\subsection{Study design and questionnaire}

The questionnaires were distributed to the randomly selected IDA registered privately practicing dentists, and were self-completed by them. The total scores of all three sections were done by examiner. The questionnaire was close ended, self assessment instrument, containing demographic details, consent form and maslach burnout inventory. The demographic details were name (optional), gender, age, qualification, specialization and professional experience.

The Burnout Syndrome's assessment was performed using the Maslach Burnout Inventory, which is an self-assessment instru- ment with answers given in Likert-type seven-point scale (0: never to 6: every day). The instrument consists of 22 questions which are divided into three dimensions, Emotional Exhaustion, depersonalization (Cynicism) and Professional achievement (Efficacy). A high score in the first two sections and a low score in the last section may indicate burnout. Validation of the MBI for use in dental practitioner was done on 40 individual.

Given that MBI is the most used instrument worldwide for this Syndrome's screening, that its metric qualities have been considered adequate and stable in several studies, and given the lack of a 'gold standard' for diagnosis of Burnout, we decided to consider the MBI as the 'gold standard' in this study, as previously recommended by Schutte et al (Denton DA et al. 2008, P.1).

\subsection{Statistical analysis}

Descriptive and inferential statistical analysis has been carried out in the present study. Results on continuous measurements are presented as Mean \pm SD (Min-Max). And results on categorical measurements are presented as percentages. Level of significance is set at 5\%. Chi-square test has been used to find the significance of study parameters on categorical scale. The Statistical software namely SPSS 15.0 was used for the analysis of the data and Microsoft word and Excel have been used to generate graphs, tables etc.

\section{Result}

Among the 150 study subjects 6 subjects i.e., $4 \%$ were below or the age of 25 years, 94 i.e., $62.7 \%$ were between $25-35$ years, 50 i.e., $33.3 \%$ were more than 35 years, (Table 1). There are 95 (63.3\%) males and $55(33.7 \%)$ females in the study population (Table 2). Around 98 (65.3\%) were with B.D.S. qualification and 52 were with M.D.S. (Table 3).

For age group, it was estimated that around $2(33.3 \%)$ participants from the $6(100 \%)$ of age group of $>25,11(11.7 \%)$ from 94 $(100 \%)$ of the $25-35$ and $4(8 \%)$ from $50(100 \%)$ were having high level of burnout.(Table 4)

Among 150 in the participant group 10 (18.2\%) females and 7 (7.4\%) maleshad high level of burnout (Table 5), which was significantly associated $(\mathrm{P}<0.05)$. It was found that $14(14.3 \%)$ with B.D.S qualification and $3(5.8 \%)$ with M.D.S. qualification were having high level of burnout. (Table 6)

Taking moderate to high in two sections and low level of one section (depersonalization), it was found around 17 (11.3\%) were having high level of burnout. (Table 7)

The overall results show, around $28.3 \%$ were moderate to high level of burnout, $46 \%$ were having moderate to high level of depersonalization, and $66.7 \%$ have low level of personal achievement. (Graph 1)

The other findings which were obtained from this study were for physical burnout. It was found that $48 \%$ were having back pain, $44.7 \%$ with headache, $34 \%$ with neck pain, , $28.7 \%$ were having hand \& wrist pain and disorder, $25.3 \%$ were suffering from cold and allergies.(Graph 2).

Table 1: Age Groups (Age Wise Distribution of Participants)

\begin{tabular}{|c|c|c|c|c|c|}
\hline & Variables & Frequency & Percent & Valid Percent & Cumulative Percent \\
\hline \multirow{4}{*}{ Valid } & $<=25 \mathrm{yrs}$ & 6 & 4.0 & 4.0 & 4.0 \\
\hline & $26-35 \mathrm{yrs}$ & 94 & 62.7 & 62.7 & 66.7 \\
\hline & $>35 \mathrm{yrs}$ & 50 & 33.3 & 33.3 & 100.0 \\
\hline & Total & 150 & 100.0 & 100.0 & \\
\hline
\end{tabular}


Table 2: Gender Groups (Gender Wise Distribution of Participants)

\begin{tabular}{llllll}
\hline & Variables & Frequency & Percent & Valid Percent & Cumulative Percent \\
\hline \multirow{3}{*}{ Valid } & Female & 55 & 36.7 & 36.7 & 36.7 \\
& Male & 95 & 63.3 & 63.3 & 100.0 \\
& Total & 150 & 100.0 & 100.0 & \\
\hline
\end{tabular}

Table 3: Qualification Group (Qualification Wise Distribution of Participants)

\begin{tabular}{llllll}
\hline & Variables & Frequency & Percent & Valid Percent & Cumulative Percent \\
\hline \multirow{3}{*}{ Valid } & BDS & 98 & 65.3 & 65.3 & 65.3 \\
& MDS & 52 & 34.7 & 34.7 & 100.0 \\
& Total & 150 & 100.0 & 100.0 & \\
\hline
\end{tabular}

Table 4: Burnout Level for Age

\begin{tabular}{|c|c|c|c|c|c|}
\hline Age in years & Burnout present & Burnout absent & Total & $\begin{array}{l}\text { Chi-square test re- } \\
\text { sult }\end{array}$ & P-value \\
\hline$<=25$ & $2(33.3 \%)$ & $4(66.3 \%)$ & $6(100 \%)$ & \multirow{4}{*}{3.455} & \multirow{4}{*}{0.178} \\
\hline $25-30$ & $11(11.7 \%)$ & $83(82.3 \%)$ & $94(100 \%)$ & & \\
\hline$>35$ & $4(8.0 \%)$ & $46(92 \%)$ & $50(100 \%)$ & & \\
\hline Total & $17(11.3 \%)$ & $133(88.7 \%)$ & $150(100 \%)$ & & \\
\hline
\end{tabular}

$\mathrm{P}<0.05$ (statistically significant), test used- chi square test.

Table 5: Burnout Level for Gender

\begin{tabular}{lllll}
\hline Gender & Burnout present & Burnout absent & Total & Chi-square test result \\
\hline Female & $10(18.2 \%)$ & $45(81.8 \%)$ & $55(100 \%)$ & \\
Male & $07(7.4 \%)$ & $88(92.6 \%)$ & $95(100 \%)$ & 4.053 \\
Total & $17(11.3 \%)$ & $133(88.7 \%)$ & $150(100 \%)$ & $0.04 *$ \\
\hline
\end{tabular}

$\mathrm{P}<0.05$ (statistically significant), test used- chi square test.

Table 6: Burnout Level for Qualifications

\begin{tabular}{lllll}
\hline Qualification & Burnout present & Burnout absent & Total & $\begin{array}{l}\text { Chi-square test } \\
\text { result }\end{array}$ \\
\hline B.D.S. & $14(14.3 \%)$ & $84(83.7 \%)$ & $55(100 \%)$ & \\
M.D.S. & $03(5.8 \%)$ & $49(94.2 \%)$ & $95(100 \%)$ & \\
Total & $17(11.3 \%)$ & $133(88.7 \%)$ & $150(100 \%)$ & \\
\hline P<0.05 (statistie & & & 0.117 \\
\hline
\end{tabular}

$\mathrm{P}<0.05$ (statistically significant), test used- chi square test.

Table 7: Total Burnout

\begin{tabular}{llllll}
\hline & High burnout & Frequency & Percent & Valid Percent & Cumulative Percent \\
\hline \multirow{3}{*}{ Valid } & Absent & 133 & 88.7 & 88.7 & 88.7 \\
& Present & 17 & 11.3 & 11.3 & 100.0 \\
& Total & 150 & 100.0 & 100.0 & \\
\hline
\end{tabular}

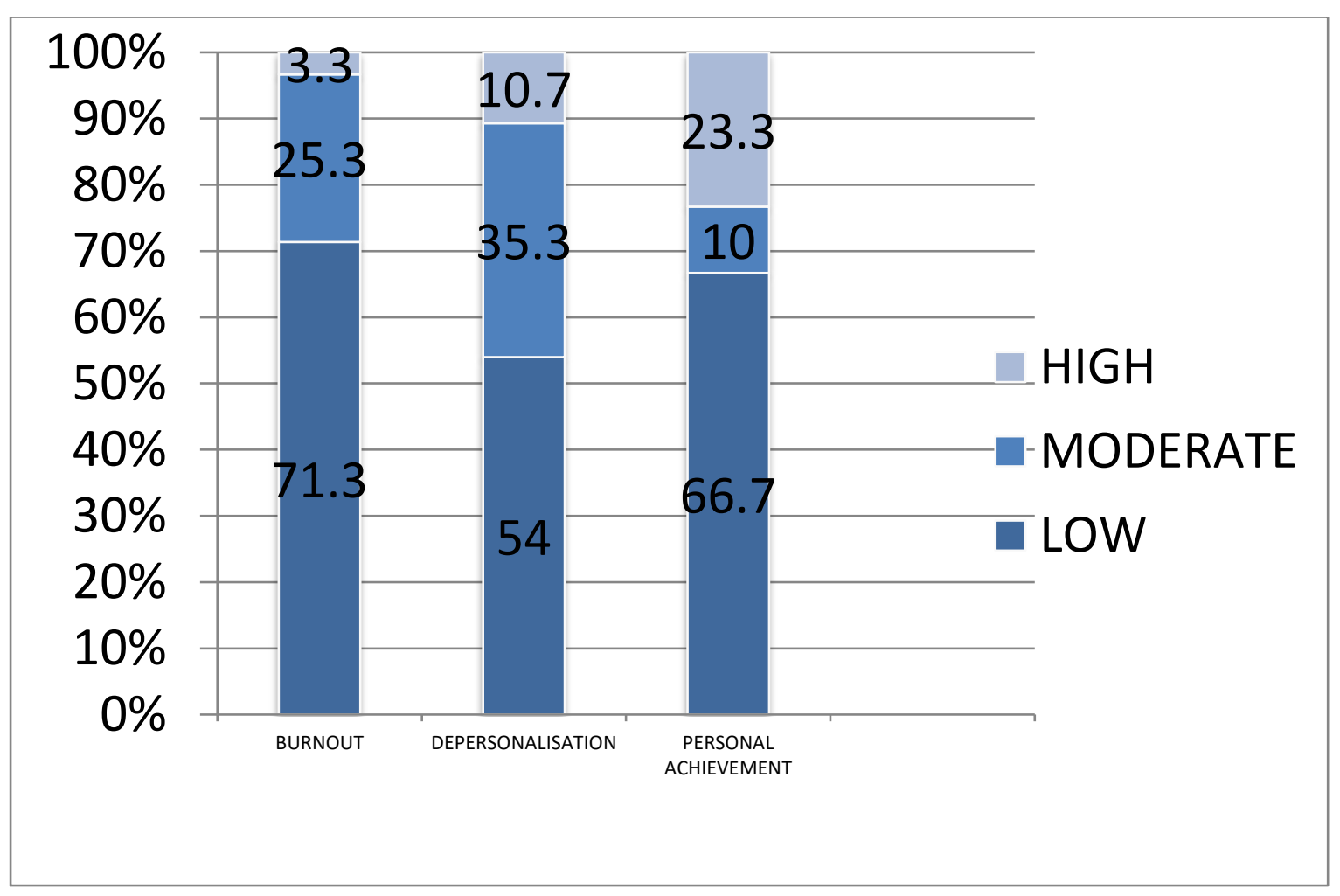




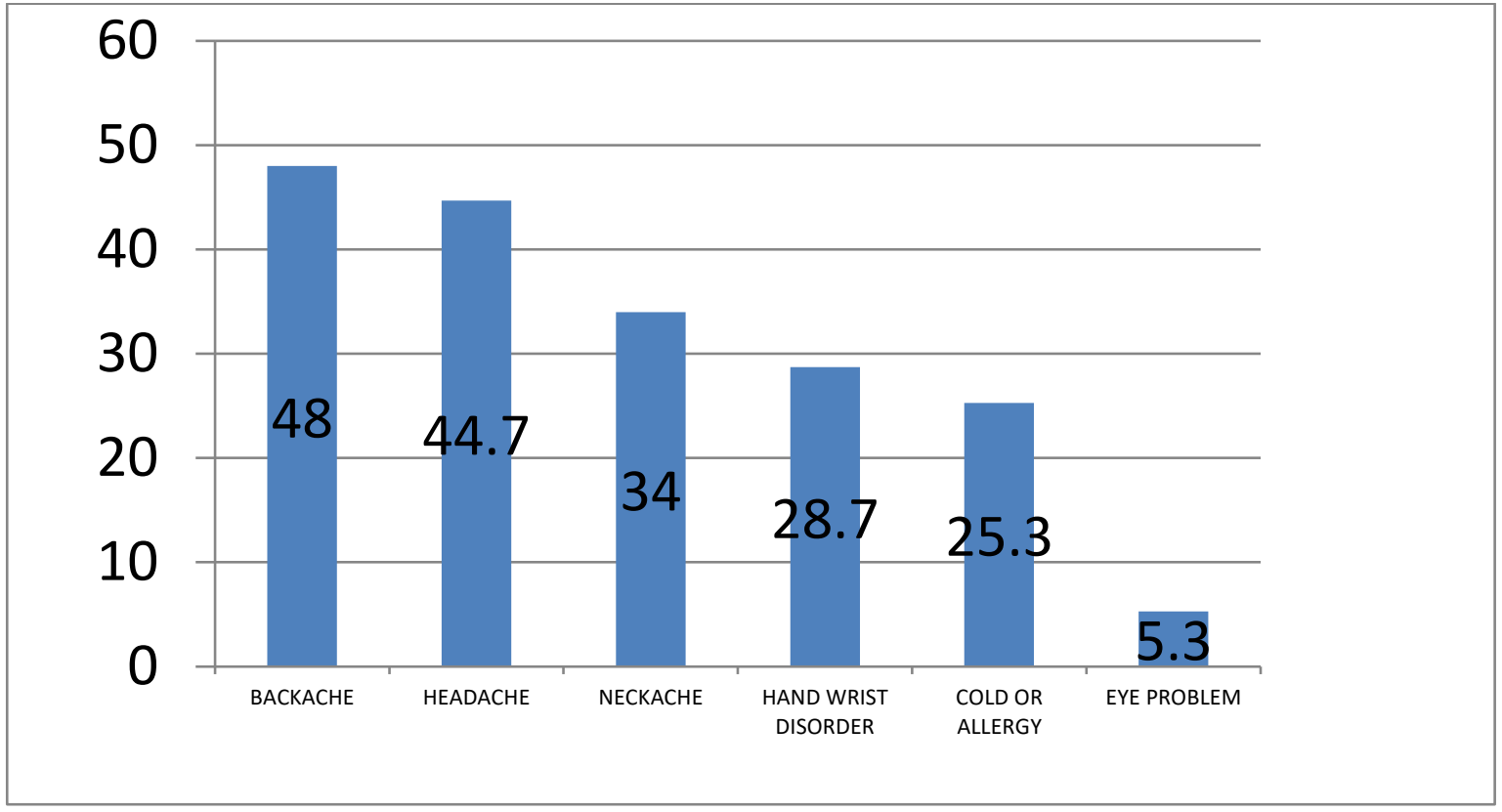

Graph 2: Distribution of Physical Burnout.

\section{Discussion}

The phenomenon of "burnout" emerged as a major social issue in the United States in the mid-1970s, and its importance has grown significantly over the past thirty years. Burnout is recognized as a potential problem within a broad range of occupations, and within many different countries (Maslach C et al 2008, p.86). Burnout poses a significant risk for dentists and should not be taken lightly. To date, only a handful of studies have investigated risks associated with increased burnout in dentists. The field of burnout is still in its infancy and much work remains to be done (Lee $\mathrm{S} \mathrm{C}$ et al 2011, p.1). That is the reason Stuart krohn called it "Burnout: The Dental Pain No One Mentions" (Krohn S 2015, p.76).

In the present study $11.3 \%$ of highburnout level found in the private practicing dentists. It is very low in number but it can lead to harmful vices like alcohol and drug addictions, extramarital affairs, and a litany of self-destructive behavior(Farran H 2012, p.12).

Similar study done in Mysore, found 11.4\% high level of burnout among private practitioner (Zucoloto $\mathrm{M}$ et al 2012, p.61)which was in accordance with study. One more study done by Gorter RC and Freeman R shows $16.8 \%$ of high burnout level in the dentist. High levels of burnout have been found, repeatedly, among 11$16 \%$ of the dentists. Burnout can thus be considered a serious risk to the dental profession, causing both a threat to the available work force and a personal tragedy for the individual. Therefore, prevention of burnout is of key importance for dental health professionals (Gorter RC et al 2012, p.87).

A study done in Brazil, Burnout syndrome was diagnosed in $32 \%$ of respondents, without statistical significant differences in the relationship among gender and working hours, marital status and years of tenure(Carneiro et al. 2013,p.266). The present study was not in accordance with the study done Brazil. This may be due to these dentists find their work stimulating and engaging, and it has been suggested that these stimulating aspects in their clinical work environment function as a buffer against burnout.(Gorter RC et al 2012, p.87, Kapoor S et al 2014, p.17). According to Maslach et al.engagement is the direct opposite of burnout. The three dimensions of engagement, namely, energy, involvement and efficacy may be viewed as the opposites of emotional exhaustion, depersonalization and diminished personal accomplishment (Basson RA 2012, p.168).

There was statistical significance found between gender and burnout association in present study $(<\mathrm{p}=0.05)$. The other finding shows relationship of burnout with age, qualification but they were not significant.
Other finding of this study shows the same results with other studies, there is higher level of burnout in age group of $<=25$ and 2535 , females, and the participants with only bachelor qualifications (Carneiro et al 2013,p.266 , Azad AA 2013,p.407). Stressfulness of work, and practice management were factors in which reality was experienced to be worse than expected. Also, finding a suitable practice was something that appeared more difficult than expected in their early career. Young dentists felt unprepared with regard to several practice management related aspects, in particular law and insurance matters, practice organization, financial management, and staff management (Gorter RC et al2007, p.279). Musculoskeletal symptoms are of major concern among dental practitioners. Work Related Musculoskeletal Disorders (WRMSD) has been reported to have a high prevalence among dentists (Sahu D et al 2015, p.307). In this study, physical exhaustion was also found out that a stressful dentist may encounter. This study shows that there was $48 \%$ were having back pain, $44.7 \%$ with headache, $34 \%$ with neck pain, , $28.7 \%$ were having hand \& wrist pain and disorder, $25.3 \%$ were suffering from cold and allergies. These results were in accordance with the study done by Veeresh DJ et al and Shahu D et al (Sahu D et al 2015, p.307, Veeresh DJ et al 2015 , p.302).Other then these findings, $44.7 \%$ headache was also found in the participants. This is one of the rare kind of study to include headache as a parameter for physical exhaustion.

The practice of dentistry involves laborious high finesse dental preparations, precision, and control in executions that require a particular attention, concentration, and patience of the dentist; and finally, the dentist's physical and mental resistance. If ergonomics principles are applied in the field of dentistry, it helps to reduce cognitive and physical stress, prevent occupational hazards, thus, helps to improve productivity and more comfort to the dentist and the patient.According to WHO and National Institute for Occupational Safety and Health, the causes of work related musculoskeletal disorder (WRMSD) are multifactorial which includes exposure to clinical condition, work place environment, organizational, psychosocial, and sociocultural variables amongst others (Sahu D et al 2015, p.307).

\section{Conclusion}

As health care provider the dentists are subjected to interpersonal stress due to the demanding nature of the occupation and close proximity to the patient. (Basson RA 2012, p.168).

Burnout does not appear abruptly, but is the final stage of a process, leading to symptoms related to the syndrome dimensions. Burnout poses a significant risk for dentists and should not be 
taken lightly. Moderate to high level of burnout were detected in the study sample, it's recommended that attention for burnout risk should be given priority by dental professionals. While the restoration of oral health and maintenance of the patient's well-being is of prime importance in the dental profession, the well-being of the service-provider should not be neglected. Additionally, it's obvious that dentists' burnout has also implications to the oral health of the patients. Hence, the problem is not only the care giver problem but also a public health problem (Basson RA 2012, p.168).

\section{Acknowledgement}

We would like to thank dept. of public health dentistry and Vydehi institute of dental science and research centre for their guidance and support. We would also like to thank all the participants of this study.

\section{References}

[1] Galán F, Ríos-Santos JV, Polo J, Rios-Carrasco B, Bullón P. Burnout, depression and suicidal ideation in dental students. Med Oral Patol Oral Cir Bucal.2013. Available on (12 December 2015). https://doi.org/10.4317/medoral.19281.

[2] Gorter RC, Freeman R. Burnout and engagement in relation with job demands and resources among dental staff in Northern Ireland. Community Dent Oral Epidemiol 2011; 39: 8795.https://doi.org/10.1111/j.1600-0528.2010.00568.x.

[3] Basson RA. Management and Prevention of Burnout in the Denta Practitioner. Dentistry 2012;3(2):168. Available on (12 December 2015). https://doi.org/10.4172/2161-1122.1000168.

[4] Zini A, Zaken Y, Ovadia-Gonen H, Mann J, Vered Y. Burnou Level among General and Specialist Dentists: A Global Manpower Concern. Occup Med Health Aff 2013;1(5):128. Available on (12 December 2015). https://doi.org/10.4172/2329-6879.1000128.

[5] Kapoor S, Puranik MP, Uma SR. Burnout in Dentistry: An Overview. Int J Adv Health Sci 2014;1(8):17-22.

[6] Denton D A, Newton J T and Bower EJ. Occupational burnout and work engagement: a national survey of dentists in the United Kingdom. British dental journal 2008:1-5. Available on http// www.ncbi.nlm.nih.gov/pubmed/18849939 ( 15 December 2015)

[7] Maslach C, Leiter M P,Schaufeli W. Measuring burnout.2008;86$108 \quad$ Available http://www.wilmarschaufeli.nl/publications/Schaufeli/179.pdf $\quad$ (15 December 2015)

[8] Lee S C et al. Risk Factors for Burnout Among Dentists: An Evidence-Based Study of the Literature.2011;1-7 Available on http//www.dentistry.utoronto.ca (21 December 2015)

[9] Krohn S. Burnout: The dental pain no one mentions 2015(6); 76-80. Available on http// www.dentaltown.com (21 December 2015)

[10] Farran H. Extinguish Burnout 2010(6); 12-14. Available on http//www.dentaltown.com (21 December 2015)

[11] Zucoloto Ml Et Al. Dentistry Teachers andthe Burnout Syndrome. Braz Dent Sci 2012 Jan-Mar; $15 \quad$ (1): 61-67. https://doi.org/10.14295/bds.2012.v15i1.729.

[12] Carneiro et al. - Burnout syndrome: evaluation in dentists in the city of Fortaleza, Brazil. RSBO 2013 Jul-Sep;10(3):266-71

[13] Azad AA. Prevalence of stress and burnout among dentists of Rawalpindi \& Islamabad Pakistan.Oral\& Dental Journal 2013 Dec; 33( 3);407-411

[14] Gorter RC, Storm MK, te Brake JM, Kersten HW and Eijkman MAJ. Outcome of career expectancies and early professional burnout among newly qualified dentists. International Dental Journal 2007; 57(4): 279-285. $\quad$ https://doi.org/10.1111/j.1875595X.2007.tb00133.x.

[15] Sahu D, Tandon S, Dhingra S, Chinmaya BR, Prasad S, Bali E, et al. Prevalence of musculoskeletal disorders among dentists: A pilot cross-sectional survey. J Indian Assoc Public Health Dent 2015; 13:307-12.https://doi.org/10.4103/2319-5932.165281.

[16] Veeresh DJ, Yunus GY, Deepta R. Prevalence of musculoskeletal pain in dental practitioners in Davangere, Karnataka: A crosssectional survey. J Indian Assoc Public Health Dent 2015; 13:3026.https://doi.org/10.4103/2319-5932.165280. 